\title{
El Peso Corporal y su Efecto sobre Otros Caracteres Morfométricos en Pollitas White Leghorn L33
}

\author{
Body Weight and its Effect on Other Morphometric Characters in \\ White Leghorn Pullets L33
}

\author{
Raiden Grandía G. ${ }^{1,3}$, Manuel Colas C. ${ }^{2}$, Julio Soroa R. ${ }^{1}$, Ángel Entrena G. ${ }^{1}$, \\ Teresa Figueroa B. ${ }^{1}$, Ana Bada B. ${ }^{1}$, Susana Jáuregui C. ${ }^{1}$, Itamys García V. ${ }^{1}$, \\ Miriam Burón R. ${ }^{1}$, Moraima Pérez G. ${ }^{1}$
}

\section{Resumen}

El objetivo del presente estudio fue determinar el efecto del peso corporal sobre otros caracteres morfométricos en pollitas White Leghorn L33. Se trabajó con 300 pollitas por seis semanas a partir de la semana 11 de edad. La mitad (grupo I) tenían el peso esperado para su edad $(865.6 \pm 13.3 \mathrm{~g})$ y la otra mitad (grupo II) estaban con sobrepeso $(920.1 \pm 37.3 \mathrm{~g})$. Se registró semanalmente el peso corporal, largo del tarso, y distancias entre pubis y de quilla a pubis. Se utilizó la prueba U de Mann-Whitney $(p<0.05)$ para comparar las medias entre grupos. La uniformidad en el peso corporal de ambos grupos fue considerada como excelente (2.6 y $4.8 \%$ para el grupo I y II, respectivamente). Se observó diferencia significativa entre grupos en el largo del tarso y la distancia de quilla a pubis ( $\mathrm{p}<0.0001)$, pero no en la distancia entre pubis. Asimismo, se observó correlación positiva entre el peso corporal y las otras variables morfométricas, aunque solo el $25.2 \%$ del largo del tarso estuvo determinado por el peso corporal. Se concluye que el peso corporal tiene un efecto directo sobre el largo del tarso en pollitas White Leghorn L33 en desarrollo, el que se potencializa en aves con sobrepeso.

Palabras clave: peso corporal, largo del tarso, distancia entre pubis, distancia de quilla a pubis, White Leghorn L33

\section{Abstract}

The aim of this study was to determine the effect of body weight on other morphometric characters in White Leghorn pullets L33. The study lasted for 6 weeks using 300 pullets (11-16 weeks old) equally distributed in two experimental groups: I: expected body weight

\footnotetext{
${ }^{1}$ Centro Nacional para la Producción de Animales de Laboratorio, CENPALAB, Cuba

${ }^{2}$ Universidad Agraria de La Habana, UNAH, Cuba

${ }^{3}$ E-mail: raiden.grandia@cenpalab.cu
}

Recibido: 15 de octubre de 2015

Aceptado para publicación: 15 de febrero de 2016 
$(865.6 \pm 13.3 \mathrm{~g})$; II: overweighed $(920.1 \pm 37.3 \mathrm{~g})$. Body weight, tarsus length and distances between pubic bones and from keel to pubis were determined weekly. The Mann-Whitney $\mathrm{U}$ test $(\mathrm{p}<0.05)$ was used to compare means between groups. Degree of uniformity in body weight in both groups was considered excellent (2.6 and $4.8 \%$ for group I and II, respectively). Significant difference between groups was observed in the tarsus length and distance from keel to pubis ( $\mathrm{p}<0.0001$ ), but not on the distance between pubic bones. Also, positive correlation between body weight and other morphometric variables was observed, although only $25.2 \%$ of the tarsus length was determined by body weight. It is concluded that body weight has a direct effect on tarsus length in White Leghorn pullets $\mathrm{L} 33$, which is potentiated in overweight birds.

Key words: body weight, tarsus length, distance between pubic bones, distance from keel to pubis, White Leghorn L33

\section{INTRODUCCIÓN}

La producción avícola en Cuba es una de las de mayor crecimiento en los últimos 30 años, lo que ha enmarcado a la isla como uno de los 45 países mayores productores de huevos a nivel mundial y entre los 25 mayores consumidores (Bejerano, 2011). En este sentido, las principales estrategias productivas en el país están dirigidas a la producción de huevos con la mayor calidad y menor costo posible (Rivera, 2013), al mismo tiempo que se estimula su desarrollo científico para la obtención de productos autóctonos sin variar la calidad exigida (FAO, 2013).

La raza White Leghorn pertenece al tronco mediterráneo, y es el resultado del cruzamiento de la línea L1 paterna con el híbrido L32 materno; de allí, la obtención del híbrido L33 con un alto nivel de puesta si la alimentación es adecuada (López et al., 1985). Son aves precoces que alcanzan la madurez sexual entre 18 y 20 semanas de edad. El peso de los huevos oscila entre 52 y $62 \mathrm{~g}$ en la etapa adulta, con una producción media de 300-305 huevos en 52 semanas (Fernández et al., 2011).

Las pollitas de reemplazo representan un animal muy estandarizado, con características bien definidas, tanto en el plano sanitario como en el zootécnico (peso, configura- ción, etc). En este sentido, Cuba ha trabajado intensamente en la caracterización de esta pollita en las etapas de inicio, crecimiento y desarrollo, particularmente en el peso corporal y largo del tarso, basado fundamentalmente en la importancia del desarrollo corporal (amplitud esquelética) y del tarso como reserva de calcio para la futura ponedora $(\mathrm{Ca}-$ rrizo y Lozano, 2007). No obstante, solo existe un trabajo que incluye el estudio de las distancias entre pubis y de quilla a pubis y su dependencia del peso corporal en las etapas de inicio y crecimiento (Lamazares et al., 2006a). Esta evaluación de la zona del pubis tiene su importancia en la estimación de la amplitud abdominal y el desarrollo del sistema reproductivo del ave ponedora (ovariooviducto), así como su preparación para el rompimiento de la postura (Schrider, 2007; Jacob et al., 2011).

La mayoría de los estudios sobre condición corporal no consideran grupos experimentales con sobrepeso, mientras que estas aves llegan a la madurez con exceso de grasa, alcanzado menores rendimientos como ponedoras que aquellas que llegan con el peso adecuado. Esta merma en la efectividad productiva se traduce en menor grosor de la cáscara del huevo, menor persistencia en la producción y mayor riesgo de muerte por prolapso del oviducto (Dottavio y Di Masso, 2010; Callejo, 2011; Martínez-Aguilar et al., 2013). Por otra parte, Pacheco (2008) menciona 
además ovulaciones erráticas y disminución de la motilidad del oviducto con producción de huevos de varias yemas y cáscara deforme.

No existen estudios en el país que analicen el efecto del peso corporal sobre el largo del tarso y el área relativa del pubis en la etapa de desarrollo en reemplazos, así como otras medidas anatómicas similares. Tampoco se dispone de información sobre la influencia del sobrepeso en estas variables morfométricas. Por ello, el presente estudio tuvo como objetivo determinar el efecto del peso corporal sobre otros caracteres morfométricos en pollitas White Leghorn L33.

\section{Materiales y Métodos}

El presente estudio se realizó en una unidad de la Empresa Avícola «Habana» que tiene como propósito garantizar el reemplazo de ponedoras White Leghorn (genotipo L33) con la crianza de pollitas en fase de desarrollo. La unidad está ubicada en el km 1.5 de la carretera de Managua, municipio Cotorro, en la provincia La Habana, Cuba. La zona se encuentra a $50 \mathrm{msnm}$, con temperatura de $23{ }^{\circ} \mathrm{C}$ y humedad relativa de $75 \%$. El experimento se llevó a cabo en febrero y marzo de 2015 y tuvo una duración de 6 semanas.

\section{Tamaño y Selección de la Muestra}

Se seleccionó una muestra de 300 pollitas de 11 semanas de edad. Las aves se distribuyeron en dos grupos experimentales: grupo I (control) formado por 150 aves con un peso promedio ideal para su edad (865.6 $\pm 13.3 \mathrm{~g}$ ) y grupo II con 150 aves con un peso promedio superior para su edad (920.1 $\pm 37.3 \mathrm{~g}$ ).

Las aves se ubicaron en una nave, colocándose en el cuartón derecho el grupo de peso regular y en el izquierdo el grupo de sobrepeso. Cada grupo constó de tres repe- ticiones (50 pollitas por repetición), separadas en hileras independientes en el centro de cada cuartón. Se realizaron observaciones e inspecciones clínicas diarias.

El estudio de aves con sobrepeso se justificó al presentarse este tipo de aves en una de las naves de la granja. Este hecho fue la consecuencia de un incremento en el suministro de alimento/ave/día en la fase de crecimiento para la recuperación de las aves con bajo peso, lo que superó en algunas pollitas los 45, 50 y 54 g establecidos como consumo para las semanas 7, 8 y 9 (Fernández et al., 2011). Sin embargo, todas las aves recibieron la misma ración de alimento con la calidad nutricional requerida para la categoría desarrollo, que incluyó entre otros componentes $14.5 \%$ de proteína bruta, $2750 \mathrm{Mcal} / \mathrm{kg}$ de peso vivo de energía metabolizable, $3.2 \%$ de grasa bruta y $2.1 \%$ de fibra bruta (Fernández et al., 2011). La ración fue suministrada por personal especializado y con gran experiencia en el manejo de aves de cría intensiva.

Asimismo, el alimento suministrado fue analizado en el Laboratorio de Control de Alimentos del CENPALAB basándose en sus características organolépticas (color, olor, textura y presencia de otros materiales), parámetros físicos (granulometría), nutritivos (materia seca, humedad, proteína, grasa y fibra brutas, cenizas, calcio y fósforo) y microbiológicos (microorganismos viables y coliformes, hongos, levaduras y Salmonella sp).

\section{Variables Morfométricas}

El peso y las medidas corporales se registraron una vez por semana. Para el peso se utilizó una balanza Salter Brecknell 235 $6 \mathrm{~S}$ con capacidad de $5 \mathrm{~kg}$ (precisión de $20 \mathrm{~g}$ ) y para las mediciones corporales se empleó un calibrador vernier.

- Peso corporal (PC). Las aves fueron pesadas (en gramos) en forma individual en el mismo horario (08:00 - 10:00), previo a la ingestión de alimento. 
- Largo del tarso (LT). Esta medida fue considerada la distancia (en $\mathrm{mm}$ ) entre la articulación metatarso-falangiana (cojinete plantar) y la articulación tibiotarsiana (extremo superior de la extremidad).

- Distancia entre pubis (PP). Se determinó como la distancia (en $\mathrm{mm}$ ) entre los extremos libres de cada hueso púbico (Schrider, 2007; Jacob et al., 2011).

- Distancia de quilla a pubis (QP). Se determinó como la distancia (en mm) entre el extremo posterior de la quilla (punta del esternón) y los extremos libres de los huesos púbicos (Schrider, 2007; Jacob et al., 2011).

- Área relativa del pubis (AP). Área resultante $\left(\mathrm{en}^{\mathrm{cm}}{ }^{2}\right)$ de la multiplicación de las distancias PP y QP (Lamazares et al., 2006b).

En la valoración de los caracteres morfométricos se consideraron como adecuados los rangos definidos para el peso corporal y largo del tarso en el Manual Tecnológico de Fernández et al. (2011), mientras que para las distancias entre pubis y de quilla a pubis se tomaron en consideración los hallazgos obtenidos por Lamazares et al. (2006a) en las etapas de inicio y crecimiento del reemplazo de ponedoras White Leghorn.

\section{Análisis Estadístico}

Se realizó una estadística descriptiva considerando valores como media, desviación estándar (DE), error estándar (EE), coeficiente de variación (CV), mínimo (Mín.) y máximo (Máx.) para el peso corporal y largo del tarso. Se determinó la uniformidad del lote en cada grupo experimental según el valor del CV del peso vivo como indicador de variabilidad $(<8 \%=$ Excelente; $8 \%=$ Bueno; $>8 \%=$ Malo) (López et al., 2010).

Se verificó si los datos seguían una distribución normal (prueba KolmogorovSmirnov) y si existía homogeneidad de varianza (prueba de Levene). Al no cumplir con estas premisas se hizo la prueba no paramétrica U de Mann-Whitney para comparar las medias de cada variable morfométrica entre grupos.

Se calcularon los coeficientes de correlación $(\mathrm{r})$ y determinación $\left(\mathrm{R}^{2}\right.$ de la regresión lineal simple) para determinar la relación directa entre las variables morfométricas. Se definió como variable independiente el peso corporal y el resto como variables dependientes. Para el análisis de los resultados se consideró como significación estadística una $\mathrm{p}<0.05$. El programa estadístico utilizado fue el SPSS v. 11.5.1.

\section{Resultados y Discusión}

La observación e inspección clínica diaria no evidenciaron manifestaciones clínicas en las aves, ni se registraron muertes. El peso inicial en la semana 11 de vida de las pollitas fue estadísticamente diferente entre los dos grupos experimentales demostrando que los grupos fueron independientes (Cuadro 1).

Las medias del peso corporal del grupo I fueron similares a los valores semanales publicados en el Manual Tecnológico de Fernández et al. (2011) para la categoría de reemplazo de ponedoras White Leghorn (Cuadro 1). Asimismo, la variabilidad del peso semanal en el grupo I osciló entre 1.5 y $3.2 \%$ (promedio de $2.6 \%$ ) y fue inferior al $4.8 \%$ de variabilidad promedio del grupo II. Según el criterio de López et al. (2010), los dos grupos presentaron una excelente uniformidad, dado que el CV fue inferior al $8 \%$.

En el trascurso de las semanas hubo un acercamiento del $\mathrm{CV}$ entre los grupos, especialmente en la semana 16 , lo que indica una mejora en la uniformidad total del lote del reemplazo producido. Este comportamiento general en la uniformidad del lote constituye una promesa de mejora en la calidad de la pollita a la edad de su incorporación a la producción de huevos. En este sentido, Lamazares et al. (2006a) y Fernández et al. 
Cuadro 1. Estadística descriptiva del peso corporal (g) de pollitas White Leghorn L33 con óptimo peso (I) y sobrepeso (II) ( $\mathrm{n}=150$ por grupo)

\begin{tabular}{clcccccc}
\hline \multirow{2}{*}{ Grupo } & \multirow{2}{*}{ Estadística $^{1}$} & \multicolumn{7}{c}{ Semana de edad } \\
\cline { 3 - 8 } & & 11 & 12 & 13 & 14 & 15 & 16 \\
\hline I & Media & 865.6 & 924.5 & 981.2 & 1051.3 & 1121.3 & 1186.8 \\
& DE & 13.3 & 22.2 & 28.6 & 30.1 & 30.9 & 37.8 \\
& EE & 1.1 & 1.8 & 2.3 & 2.5 & 2.5 & 3.1 \\
& CV (\%) & 1.5 & 2.4 & 2.9 & 2.9 & 2.8 & 3.2 \\
& Mín. & 840 & 900 & 940 & 980 & 1040 & 1080 \\
& Máx. & 880 & 960 & 1020 & 1100 & 1180 & 1240 \\
& Media & 920.1 & 977.2 & 1033.2 & 1093.1 & 1161.2 & 1237.9 \\
& DE & 37.3 & 52.9 & 54.7 & 52.0 & 55.5 & 54.1 \\
& EE & 3.0 & 4.3 & 4.5 & 4.3 & 4.5 & 4.4 \\
& CV (\%) & 4.1 & 5.4 & 5.3 & 4.8 & 4.8 & 4.4 \\
& Mín. & 880 & 900 & 940 & 1000 & 1060 & 1140 \\
& Máx. & 1020 & 1080 & 1140 & 1180 & 1260 & 1340 \\
\hline
\end{tabular}

${ }^{1}$ DE: desviación estándar; EE: error estándar; CV: coeficiente de variación; Mín.: mínimo; Máx.: máximo

(2011) refieren que al trabajar en función de lograr los pesos requeridos en el reemplazo con el desarrollo esquelético y la uniformidad adecuada se logra una entrada temprana y pareja en la producción, un buen desarrollo de la curva de producción, alto pico de puesta, mejor tamaño y calidad de los huevos producidos, unido a una buena persistencia y una alta viabilidad.

Según Itza et al. (2011) y TapiaArguelles et al. (2014), el largo del tarso es un indicador de crecimiento corporal y medida distintiva entre especies aviares. En el presente estudio se observó un incremento paralelo entre el peso corporal y largo del tarso con la edad (Cuadros 1 y 2). En el grupo II se obtuvieron valores medios superiores y mayor variabilidad en el largo del tarso en comparación con el grupo I, cuyas medidas fueron adecuadas para su edad (Cuadro 2). Según Fernández et al. (2011), el largo del tarso de las pollitas en la semana 16 debió ser $103 \mathrm{~mm}$, pero fue de $106.6 \mathrm{~mm}$ en el grupo II, valor superior a los $104 \mathrm{~mm}$ esperados para la semana 18 , que es cuando se planifica la incorporación del reemplazo a la prepostura.

La relación observada entre el peso corporal y el largo del tarso está condicionada fundamentalmente por ser indicadores de crecimiento y desarrollo corporal en las aves, variables que incrementan simultáneamente no solo en líneas ligeras, sino también en reproductoras pesadas (Quintana, 2001). Asimismo, ha sido demostrada una correlación positiva media entre estas dos variables morfométricas en la gallina Criolla cubana (Pérez et al., 2004).

En el Cuadro 3 se observó una tendencia al incremento del valor promedio de las variables analizadas, lo que puede demostrar que estos caracteres morfométricos constituyen indicadores de crecimiento y desarro- 
Cuadro 2. Estadística descriptiva del largo del tarso $(\mathrm{mm})$ de pollitas White Leghorn L33 con óptimo peso (I) y sobrepeso (II) ( $\mathrm{n}=150$ por grupo)

\begin{tabular}{cccccccc}
\hline \multirow{2}{*}{ Grupo } & Estadística & \multicolumn{7}{c}{ Semana de edad } \\
\cline { 3 - 8 } & I & 11 & 12 & 13 & 14 & 15 & 16 \\
\hline & Media & 95.3 & 97.9 & 100.1 & 101.3 & 102.3 & 103.3 \\
& DE & 2.4 & 2.4 & 2.5 & 2.6 & 2.6 & 2.6 \\
& EE & 0.2 & 0.2 & 0.2 & 0.2 & 0.2 & 0.2 \\
& CV (\%) & 2.5 & 2.4 & 2.5 & 2.5 & 2.5 & 2.5 \\
& Mín. & 91 & 93 & 95 & 96 & 97 & 98 \\
& Máx. & 103 & 105 & 107 & 109 & 110 & 111 \\
& Media & 98.1 & 100.8 & 103.1 & 104.6 & 105.6 & 106.6 \\
& DE & 4.2 & 4.4 & 4.4 & 4.3 & 4.3 & 4.3 \\
& EE & 0.4 & 0.4 & 0.4 & 0.4 & 0.4 & 0.4 \\
& CV (\%) & 4.3 & 4.4 & 4.3 & 4.1 & 4.1 & 4.1 \\
& Mín. & 90 & 92 & 93 & 94 & 95 & 96 \\
& Máx. & 103 & 106 & 109 & 110 & 111 & 112 \\
\hline
\end{tabular}

${ }^{1} \mathrm{DE}$ : desviación estándar; EE: error estándar; $\mathrm{CV}$ : coeficiente de variación; Mín.: mínimo; Máx.: máximo

Cuadro 3. Comparación de medias de variables morfométricas en pollitas White Leghorn L33 con óptimo peso (I) y sobrepeso (II) ( $\mathrm{n}=150$ por grupo)

\begin{tabular}{cccccccc}
\hline & Grupo & \multicolumn{5}{c}{ Semana de edad } \\
\cline { 3 - 7 } & & 11 & 12 & 13 & 14 & 15 & 16 \\
\hline PC $(\mathrm{g})$ & I & $865.6^{\mathrm{a}}$ & $924.5^{\mathrm{a}}$ & $981.2^{\mathrm{a}}$ & $1051.3^{\mathrm{a}}$ & $1121.3^{\mathrm{a}}$ & $1186.8^{\mathrm{a}}$ \\
& II & $920.1^{\mathrm{b}}$ & $977.2^{\mathrm{b}}$ & $1033.2^{\mathrm{b}}$ & $1093.1^{\mathrm{b}}$ & $1161.2^{\mathrm{b}}$ & $1237.9^{\mathrm{b}}$ \\
LT $(\mathrm{mm})$ & I & $95.3^{\mathrm{a}}$ & $97.9^{\mathrm{a}}$ & $100.1^{\mathrm{a}}$ & $101.3^{\mathrm{a}}$ & $102.3^{\mathrm{a}}$ & $103.3^{\mathrm{a}}$ \\
& II & $98.1^{\mathrm{b}}$ & $100.8^{\mathrm{b}}$ & $103.1^{\mathrm{b}}$ & $104.6^{\mathrm{b}}$ & $105.6^{\mathrm{b}}$ & $106.6^{\mathrm{b}}$ \\
PP (mm) & I & $11.9^{\mathrm{a}}$ & $11.9^{\mathrm{a}}$ & $13.9^{\mathrm{a}}$ & $13.9^{\mathrm{a}}$ & $16.4^{\mathrm{a}}$ & $16.4^{\mathrm{a}}$ \\
& II & $12.7^{\mathrm{a}}$ & $12.7^{\mathrm{a}}$ & $14.7^{\mathrm{a}}$ & $14.7^{\mathrm{a}}$ & $17.2^{\mathrm{a}}$ & $17.2^{\mathrm{a}}$ \\
QP $(\mathrm{mm})$ & I & $18.9^{\mathrm{a}}$ & $18.9^{\mathrm{a}}$ & $20.9^{\mathrm{a}}$ & $20.9^{\mathrm{a}}$ & $23.4^{\mathrm{a}}$ & $23.4^{\mathrm{a}}$ \\
& II & $23.4^{\mathrm{b}}$ & $23.4^{\mathrm{b}}$ & $25.4^{\mathrm{b}}$ & $25.4^{\mathrm{b}}$ & $27.9^{\mathrm{b}}$ & $27.9^{\mathrm{b}}$ \\
AP $\left.(\mathrm{cm})^{2}\right)$ & I & $2.2^{\mathrm{a}}$ & $2.2^{\mathrm{a}}$ & $2.9^{\mathrm{a}}$ & $2.9^{\mathrm{a}}$ & $3.8^{\mathrm{a}}$ & $3.8^{\mathrm{a}}$ \\
& II & $3.0^{\mathrm{b}}$ & $3.0^{\mathrm{b}}$ & $3.7^{\mathrm{b}}$ & $3.7^{\mathrm{b}}$ & $4.8^{\mathrm{b}}$ & $4.8^{\mathrm{b}}$ \\
\hline
\end{tabular}

PC: peso corporal; LT: largo del tarso; PP: distancia entre pubis; QP: distancia de quilla a pubis; AP: área relativa del pubis

${ }^{a, b}$ Superíndices diferentes entre grupos y dentro de semanas de edad indican diferencia estadística $(p<0.0001)$ 
Cuadro 4. Relación del peso corporal con otras variables morfométricas ${ }^{1}$ de 300 pollitas White Leghorn L33

\begin{tabular}{cccccc}
\hline $\begin{array}{c}\text { Semana de } \\
\text { edad }\end{array}$ & $\begin{array}{c}\text { Variable } \\
\text { dependiente }\end{array}$ & $\mathrm{r}$ & $\mathrm{R}^{2}$ & $\mathrm{EE}$ & $\mathrm{p}$ \\
\hline \multirow{4}{*}{11} & LT & 0.47 & 21.6 & 0.0048 & $<0.0001$ \\
& PP & 0.18 & 3.2 & 0.0053 & 0.002 \\
& QP & 0.20 & 4.1 & 0.0100 & $<0.0001$ \\
& AP & 0.26 & 6.8 & 0.0018 & $<0.0001$ \\
\hline \multirow{4}{*}{12} & LT & 0.46 & 21.4 & 0.0038 & $<0.0001$ \\
& PP & 0.16 & 2.6 & 0.0043 & 0.005 \\
& QP & 0.13 & 1.7 & 0.0081 & 0.026 \\
& AP & 0.19 & 3.7 & 0.0014 & 0.001 \\
\hline \multirow{4}{*}{13} & LT & 0.50 & 25.2 & 0.0038 & $<0.0001$ \\
& PP & 0.15 & 2.4 & 0.0041 & 0.008 \\
& QP & 0.11 & 1.1 & 0.0078 & 0.065 \\
& AP & 0.17 & 2.9 & 0.0015 & 0.003 \\
\hline \multirow{3}{*}{16} & LT & 0.47 & 21.9 & 0.0042 & $<0.0001$ \\
& PP & 0.14 & 1.9 & 0.0044 & 0.017 \\
& QP & 0.04 & 0.2 & 0.0084 & 0.478 \\
& AP & 0.12 & 1.3 & 0.0017 & 0.048 \\
\hline & LT & 0.43 & 18.4 & 0.0041 & $<0.0001$ \\
& PP & 0.13 & 1.6 & 0.0042 & 0.027 \\
& QP & 0.01 & 0 & 0.0081 & 0.848 \\
& AP & 0.08 & 0.7 & 0.0018 & 0.145 \\
\hline \multirow{3}{*}{15} & LT & 0.43 & 18.6 & 0.0038 & $<0.0001$ \\
& PP & 0.15 & 2.2 & 0.0039 & 0.010 \\
& QP & 0.06 & 0.3 & 0.0075 & 0.332 \\
& AP & 0.13 & 1.8 & 0.0017 & 0.022 \\
\hline
\end{tabular}

${ }^{1}$ LT: largo del tarso; PP: distancia entre pubis; QP: distancia de quilla a pubis; AP: área relativa del pubis

r: Coeficiente de correlación; $\mathrm{R}^{2}$ : Coeficiente de determinación

llo de las pollitas (Lamazares et al., 2006a,b). Asimismo, se pudo observar diferencias significativas entre grupos por semana para peso corporal, largo del tarso, distancia de quilla a pubis y área relativa del pubis $(\mathrm{p}<0.0001)$; sin embargo, no se evidenciaron diferencias entre grupos con relación a la distancia entre pubis.
Las aves del grupo II presentaron mayor dimensión del área relativa del pubis $(p<0.0001)$ en todas las semanas del estudio (Cuadro 3), lo que indica una mayor amplitud abdominal de la futura gallina. Asimismo, supone una estimación mayor del crecimiento y maduración del aparato reproductor y del tamaño del hígado, órgano que se prepara 
para el rompimiento de la postura (Schrider, 2007; Jacob et al., 2011). Esta inferencia coincide con lo expresado por Lamazares et al. (2006b), quienes concluyeron que las medidas y peso del huevo están determinados en gran medida por el peso corporal. Sin embargo, esta condición de sobrepeso promete en otro sentido, rendimientos productivos inferiores en las futuras ponedoras, ya que suele observarse menor grosor de la cáscara del huevo, haciéndolos más quebradizos, así como menor persistencia en la producción y mayor riesgo de muerte por prolapso del oviducto (Dottavio y Di Masso, 2010; Callejo, 2011; Martínez-Aguilar et al., 2013). También, Pacheco (2008) refiere como consecuencia de ello la ovulación errática y disminución de la motilidad del oviducto con producción de huevos de varias yemas y cáscaras deformes.

El análisis del coeficiente de correlación evidenció de manera general una correlación positiva entre el peso corporal y las otras variables morfométricas en las 6 semanas del estudio (Cuadro 4). Sin embargo, solo existió una correlación positiva media entre el peso corporal y el largo del tarso (0.43-0.50), a diferencia del resto de las variables que presentaron correlaciones bajas con el peso corporal $(0.01-0.26)$.

Estos hallazgos coinciden con Pérez et al. (2004) y Lamazares et al. (2006a) quienes demostraron una mayor influencia del peso vivo sobre el largo del tarso, pero no así sobre las distancias entre pubis y de quilla a pubis, indicando la influencia de otros factores determinantes. En contraste, Babaahmady et al. (2010) encontraron que las distancias de quilla a pubis y entre los huesos púbicos están determinadas por el peso corporal.

El coeficiente de determinación evidenció de manera general que la variabilidad del largo del tarso está determinada entre un 18.4 y $25.2 \%$ por la variabilidad del peso corporal (Cuadro 4), mientras que se observaron va- lores inferiores en la determinación sobre el área relativa del pubis y la distancia entre pubis. Sin embargo, solamente en las semanas 11 y 12 se demostró que la variabilidad de la distancia de quilla a pubis estuvo determinada por el peso corporal $(p<0.05)$. En este sentido, los resultados obtenidos coinciden con Lamazares et al. (2006a) quienes encontraron en las fases de inicio y crecimiento una baja relación entre el peso corporal y las distancias entre pubis y de quilla a pubis, lo que infiere una mayor influencia de otros factores determinantes como la genética.

Se concluye que el peso corporal tiene un efecto directo sobre el largo del tarso en pollitas White Leghorn L33 en desarrollo, el que se potencializa bajo la condición de sobrepeso.

\section{Literatura Citada}

1. Babaahmady E, Ghorani M, Kurdavan R. 2010. Morphometric evaluation of variables measurement chickens for replacement of egg-laying hens. Iran J Vet Clin Sci 4: 121-131.

2. Bejerano G. 2011. Evaluación del sistema de alojamiento para gallinas ponedoras White Leghorn L-33 en condiciones de producción comercial. Rev Cub Cienc Avícola 35: 69-84.

3. Callejo A. 2011. Alimentación y control de peso en las futuras ponedoras. OpenCourseWare [Internet]. Disponible en: http://ocw.upm.es/produccion-animal/ produccion-avicola/contenidos/ TEMA_5/5-3-alimentacion-y-controlde-peso-en-las-futuras-ponedoras/view

4. Carrizo J, Lozano J. 2007. Alimentación de las pollitas e inicio de puesta. XXIII Curso de Especialización FEDNA. [Internet]. Disponible en: http:/ /www.produccion-animal.com.ar/ produccion_aves/produccion_avicola/ 71-07CAP_V.pdf 
5. Dottavio A, Di Masso R. 2010. Mejoramiento avícola para sistemas productivos semi-intensivos que preservan el bienestar animal. BAG J Basic Appl Genet 21: 2-10.

6. Fernández A, Madrazo G, Bermúdez J, Pérez M, González R, López S, Bacallao A. 2011. Ponedoras y sus reemplazos. En: Manual tecnológico para la cría de aves. La Habana, Cuba: Instituto de Investigaciones Avícolas. p 72-77.

7. [FAO] Organización de las Naciones Unidas para la Alimentación y la Agricultura. 2013. Revisión del desarrollo avícola. FAO [Internet]. Disponible en: http://www.fao.org/docrep/019/ i3531s/i3531s.pdf

8. Itza O, Ortiz O, Janacua V, Olguien A, Quintero E, Rodríguez A, Martín O. 2011. Características de crecimiento de pollitas de postura en relación al tipo de alojamiento. Pesq Agropec Bras 46: 768-771. doi: 10.1590/S0100204X2011000700013

9. Jacob J, Pescatore T, Cantor A. 2011. Evaluating egg laying hens. Premier One Supplies [Internet]. Disponible en: https:/ /www.premier 1 supplies.com/img/ newsletter/02-27-14-poultry/ Evaluating_egg_laying_hens.pdf

10. Lamazares M, Hernández $O$, Soto $E$, Oviedo M. 2006a. Estudio morfométrico del inicio - reemplazo de ponedoras. REDVET 7(10). [Internet]. Disponible en: http://www.veterinaria.org/revistas/redvet/n101006/ 100614.pdf

11. Lamazares $M$, Hernández $O$, Nodarse L, Díaz L. 2006b. Influencia del tamaño del pubis y el peso de la ponedora en el tamaño y peso de los huevos. REDVET 7(10). [Internet]. Disponible en: http://www.veterinaria.org/ revistas/redvet/n101006/100613.pdf

12. López A, García MCS, Sánchez AP. 2010. La industria avícola. En: Sánchez A, López A, García MCS, et al. (eds).
Salud y producción de las aves. $3^{\mathrm{a}}$ ed. La Habana, Cuba: Ed Félix Varela. p 134.

13. López A, Pinillos M, Pérez E. 1985. Manual de teoría, cría y explotación de las aves. La Habana, Cuba: EMPES. 318 p.

14. Martínez-Aguilar Y, López-Figueredo Y, Martínez-Yero O, Olmo-González $C$, Rodríguez-Bertot $R$. 2013. Influencia del peso vivo de gallinas ponedoras White Leghorn (L33) en la producción y calidad del huevo comercial. Rev Granma Ciencia 17: a8. [Internet]. Disponible en: http://www.grciencia. granma.inf.cu/2013_17_n1_a8.html

15. Pacheco V. 2008. Complicaciones del sobrepeso en gallinas reproductoras pesadas. SIRIVS. [Internet]. Disponible en: http://veterinaria.unmsm.edu.pe/files/ Articulo_pacheco_Final.pdf

16. Pérez A, Polanco G, Pérez Y. 2004. Algunas características morfológicas del exterior de la gallina local de la región central de la provincia de Villa Clara, Cuba. Livestock Res Rural Dev 16(10). [Internet]. Disponible en: http:// www.lrrd.org/lrrd16/10/pere16076.htm

17. Quintana J. 2001. Efecto de la proteína cruda, energía metabolizable y consumo de alimento sobre la productividad, incubabilidad, contenido de lípidos en suero, huevo y hormonas sexuales en gallinas reproductoras pesadas. Tesis de Doctorado. Colima, México: Universidad de Colima. $72 \mathrm{p}$.

18. Rivera MSC. 2013. Peligro microbiológico en alimentos de importancia en Cuba. En: Pérez M, Pérez L, Urquiaga I, et al. (eds). El análisis de riesgos como base de los sistemas de inocuidad de los alimentos. La Habana, Cuba: Centro de Gestión y Desarrollo de la Calidad (CGDC). p 154-155.

19. Schrider D. 2007. Selecting for egg production. American Livestock Breeds Conservancy [Internet]. Disponible en: http://www.livestockconservancy.org/ i m a g e s / u p loa d s / d o c s / ALBCchicken_assessment-2.pdf 
20. Tapia-Arguelles L, Mazorra-Calero C, Jiménez-Fernández R, Fole-Cruz E, González-González A. 2014. Comportamiento productivo de gallinas ponedoras White Leghorn suplementa- das con frutos frescos de Morinda citrifolia (noni). Rev Granma Ciencia 18: a7. [Internet]. Disponible en: http:// www.grciencia. granma.inf.cu/ 2014_18_n2_a7.html 